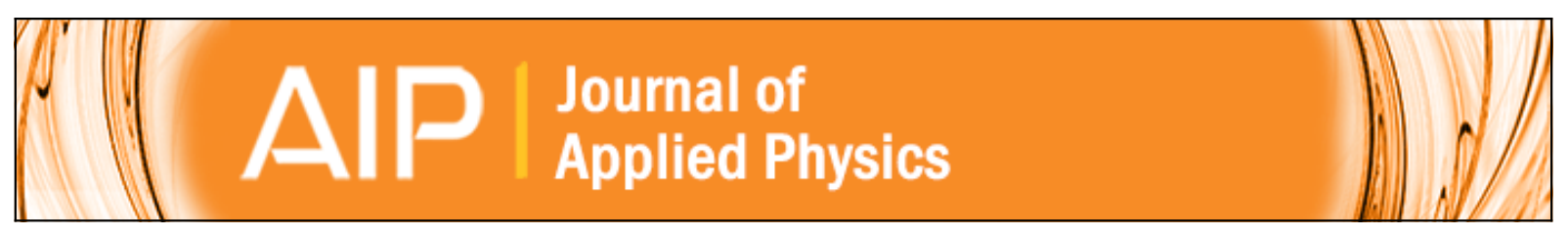

\title{
Electromagnetic heating effect of aggregated gold nanoparticle colloids
}

Xiaoming Liu, Hui-jiuan Chen, Xiaodong Chen, Yasir Alfadhl, Junsheng Yu, and Dongsheng Wen

Citation: Journal of Applied Physics 115, 094903 (2014); doi: 10.1063/1.4867615

View online: http://dx.doi.org/10.1063/1.4867615

View Table of Contents: http://scitation.aip.org/content/aip/journal/jap/115/9?ver=pdfcov

Published by the AIP Publishing

\section{Articles you may be interested in}

Targeting mitochondria in cancer cells using gold nanoparticle-enhanced radiotherapy: A Monte Carlo study Med. Phys. 42, 1119 (2015); 10.1118/1.4906192

Effects of core/shell structure on magnetic induction heating promotion in $\mathrm{Fe} 3 \mathrm{O} 4 / \mathrm{\gamma}-\mathrm{Fe} 2 \mathrm{O} 3$ magnetic nanoparticles for hyperthermia

Appl. Phys. Lett. 103, 163104 (2013); 10.1063/1.4825270

Radiosensitizing effect of gold nanoparticles in carbon ion irradiation of human cervical cancer cells AIP Conf. Proc. 1530, 205 (2013); 10.1063/1.4812924

A stochastic model of cell survival for high-Z nanoparticle radiotherapy

Med. Phys. 40, 024102 (2013); 10.1118/1.4773885

Enhanced relative biological effectiveness of proton radiotherapy in tumor cells with internalized gold nanoparticles

Appl. Phys. Lett. 98, 193702 (2011); 10.1063/1.3589914

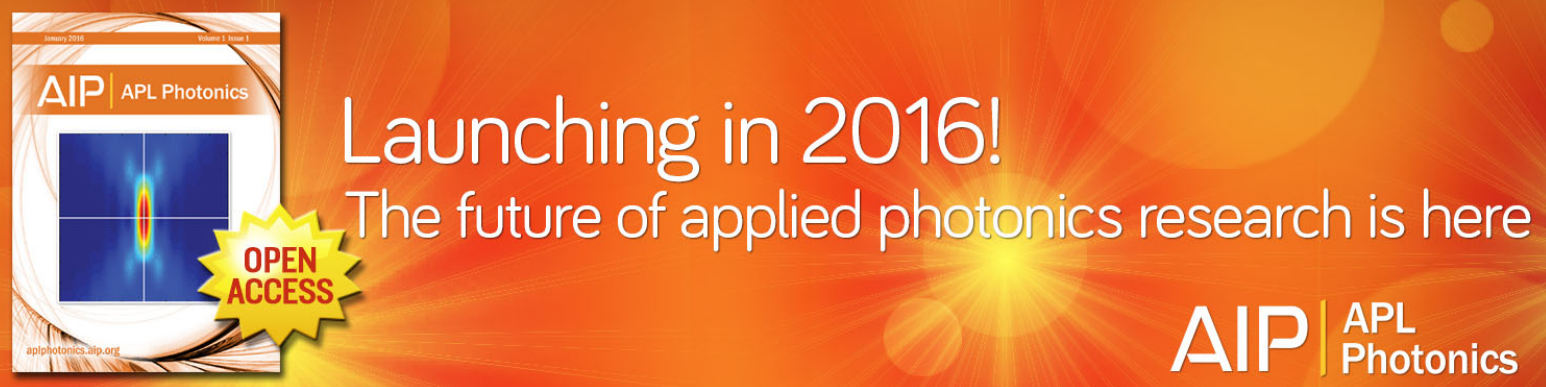




\title{
Electromagnetic heating effect of aggregated gold nanoparticle colloids
}

\author{
Xiaoming Liu, ${ }^{1,2, a)}$ Hui-jiuan Chen, ${ }^{3}$ Xiaodong Chen, ${ }^{2}$ Yasir Alfadhl, ${ }^{2}$ Junsheng Yu, ${ }^{1}$ \\ and Dongsheng Wen ${ }^{3,4, b)}$ \\ ${ }^{1}$ International Joint Lab on EM Theory and Applications, Beijing Key Laboratory of Work Safety \\ Intelligent Monitoring, School of Electronic Engineering, Beijing University of Posts and Telecommunications, \\ No. 10, Xi-Tu-Cheng Road, Haidian District, Beijing, China, 100876 \\ ${ }^{2}$ School of Electronic Engineering and Computer Science, Queen Mary University of London, London, \\ United Kingdom \\ ${ }^{3}$ School of Materials Science and Engineering, Queen Mary University of London, London, United Kingdom \\ ${ }^{4}$ School of Process, Environmental and Materials Engineering, University of Leeds, Leeds, United Kingdom
}

(Received 8 January 2014; accepted 22 February 2014; published online 4 March 2014)

\begin{abstract}
Gold nanoparticles (GNPs) have been recently investigated intensively for potential hyperthermia treatment of malignant cancer cells in combination with radiofrequency (RF) electromagnetic (EM) fields/waves. However, many controversial results have been reported on whether GNPs can be heated by EM fields. It has been suggested that aggregated GNPs may be heated significantly by a RF field, which however has not been examined experimentally. This work proposes a novel electric treatment of mono-dispersed particles to create aggregated GNPs, and conducts an investigation of their bulk heating behavior under a $655 \mathrm{~nm}$ laser and a $13.56 \mathrm{MHz} \mathrm{RF}$ electric field. It is revealed that the heating rates of aggregated colloids are significantly higher than those of mono-dispersed GNPs for the $655 \mathrm{~nm}$ laser, whereas at $13.56 \mathrm{MHz}$, the heating effects are barely noticeable for both aggregated and mono-dispersed colloids. Various possible reasons are discussed and the negligible electric field enhancement is believed to be responsible at $13.56 \mathrm{MHz}$. (C) 2014 AIP Publishing LLC. [http://dx.doi.org/10.1063/1.4867615]
\end{abstract}

\section{INTRODUCTION}

Gold nanoparticles (GNPs) are currently under intensive investigation for biomedical applications, such as bioimaging, ${ }^{1,2}$ drug delivery, ${ }^{3,4}$ and cancer treatment, ${ }^{5,6}$ due to their good biocompatibility and soft chemical properties, which facilitate their conjugation with ligands and biomolecules. Recently, GNPs have been proposed for non-invasive radiofrequency (RF) hyperthermia treatment of cancer. ${ }^{7-15}$ The basic idea of this hypothesis includes a few steps: (i) GNPs coated with peptides or molecules that can detect cancer cells are injected into cancerous tissue; (ii) After GNPs attaching on the membrane of cancer cells, RF fields are applied to tissues incubated with GNPs; and (iii) GNPs absorb RF energy and heat up, inducing the hyperthermia effect. It is normally required that the regional high temperature needs to be $>42{ }^{\circ} \mathrm{C}$ to cause any lethal effect to cancer cells. ${ }^{16}$

Nevertheless, there are many controversies and uncertainties existing in the published work regarding whether GNPs can be heated by RF fields. Initially, Gannon et al. ${ }^{7}$ reported that GNP colloids could be heated much more rapidly than the base fluid, i.e., de-ionized water (DI Water). Moran ${ }^{9}$ reported a size-dependent heating effect of GNP colloids and attributed this phenomenon to the Joule heating. Later on, $\mathrm{Li}^{13}$ and $\mathrm{Liu}^{14}$ experimentally reported that the heating effect of GNP colloids was not due to the presence of GNPs, but the ionic impurities within the colloid. Theoretically, Hanson ${ }^{17}$ investigated the heating mechanisms of GNPs from various aspects, including the dielectric

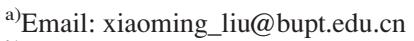

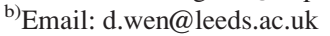

loss, phonon transfer, and the surface electron effect, and concluded that naked GNPs could not be heated by $13.56 \mathrm{MHz}$ RF field. Similarly, another theoretical work by Sassaroli ${ }^{18}$ also concluded that naked GNPs could hardly be heated by low frequency RF fields. However, it was suggested that charged GNPs could be heated through the electrophoresis phenomenon under a cyclic electric field. Subsequently, Stuart $^{19}$ investigated the heating of citrate coated GNPs under 13.56 MHz, which indeed showed some heating effects.

The electrophoretic effect in GNP incubated tissues is expected to be small in practical applications since the mobility of GNPs is limited in the tissue. However, experimentally, Gannon ${ }^{7}$ reported that GNP incubated tissue could be heated under $13.56 \mathrm{MHz}$, resulting in the temperature increase of the malignant tissue by several centigrade. Clearly, this heating effect could not be due to mono-dispersed naked GNPs, whose effects have been disproved both theoretically ${ }^{17,18}$ and experimentally. ${ }^{13,14}$ There shall have other mechanisms responsible for the reported temperature rise. A lot of efforts have been made to understand the heating process of GNPs, either pure GNPs or coated/charged GNPs, in order to produce a controlled heating effect, ${ }^{20-22}$ which still lacks the consistency. The reasons are multi-folds, which may include: (i) the existence of many possible mechanisms; (ii) the idealized assumptions for all theoretical models; and (iii) the limitation of the experiment accuracy, which prevents the differentiation of competing mechanisms from each other.

In this regard, it is essential to investigate the heating mechanisms separately, particularly mimicking the situation when tissues are incubated with GNPs. One of the mechanisms that has been mentioned in both Refs. 17 and 18, but not yet addressed, is the effect of GNP aggregation. 
Aggregation of GNPs is very likely to happen when ligands coated GNPs are injected to target cancer cells. ${ }^{7}$ It has been reported that aggregated particles can increase the absorption cross section in the IR region. ${ }^{23,24}$ However, whether this happens at $13.56 \mathrm{MHz}$ requires accurate experimental verification. One difficulty lies in the production of aggregated GNPs without changing the volume ratio. To investigate this issue properly, this study reports a novel electric field aided method to prepare aggregated GNPs while keeping the volume ratio unchanged. Then the aggregated GNP colloids are heated under a laser at $650 \mathrm{~nm}$ wavelength and a RF field at 13.56 MHz. Such a comparative study would allow the verification of reported results at high electromagnetic (EM) frequency, ${ }^{23,24}$ and the investigation of the heating contribution from aggregated particles at a low RF frequency.

The paper is organized as follows: Section II briefly describes the procedure of GNP synthesis; Section III reports the procedure of the preparation of aggregated GNPs; Sections IV and V present the heating results of the $650 \mathrm{~nm}$ laser and 13.56 MHz RF field; and Section VI concludes this work.

\section{PREPARATION OF MONO-DISPERSED GOLD NANOPARTICLES}

Gold nanoparticles were synthesized by the citrate reduction method with the aid of ultrasonication. ${ }^{25}$ In brief, $5.0 \times 10^{-5} \mathrm{~mol}_{\text {of }} \mathrm{HAuCl}_{4}$ in $190 \mathrm{ml}$ of DI water was heated until boiling. While the solution was kept heated and stirred by a magnetic blender, $10 \mathrm{ml}$ of $0.5 \%$ sodium citrate was added. The solution was kept stirring for the next $30 \mathrm{~min}$ until the reaction was complete. Other than GNPs, some residual substances, such as $\mathrm{Na}^{+}, \mathrm{Cl}^{-}$, and $\mathrm{C}_{5} \mathrm{H}_{6} \mathrm{O}_{5}$, also exist in the colloid. Gold colloids were purified by the membrane dialysis method. In this process, $100 \mathrm{ml}$ of GNP colloid was put in a membrane tube with pore size of 2-3 nm in diameter, which allows the smooth diffusion of ions but keeps GNPs inside. The membrane was located in a flask of DI water $\sim 2000 \mathrm{ml}$, stirred by a magnetic stirrer. The DI water was changed twice per day and the purification process lasted for 5 days. During this process, the concentrations of various impurities diminished exponentially with the times of DI water change, which resulted in a negligible impurities presence in the colloid and confirmed by the UV-Vis spectrum measurement. The GNP colloids after the purification process are therefore termed as "purified sample" in this study. The processes are illustrated in Fig. 1.

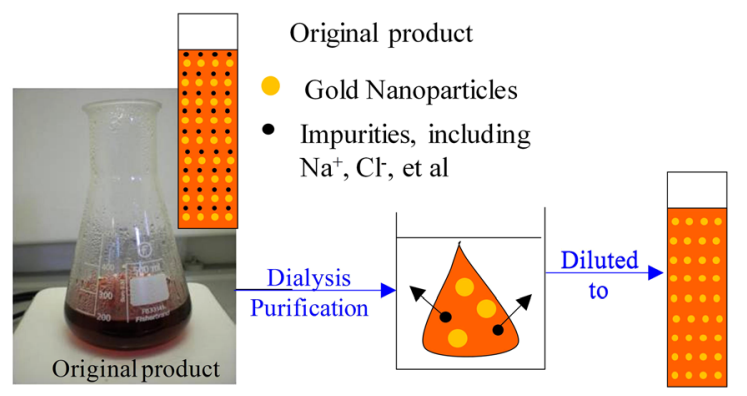

Purified samples

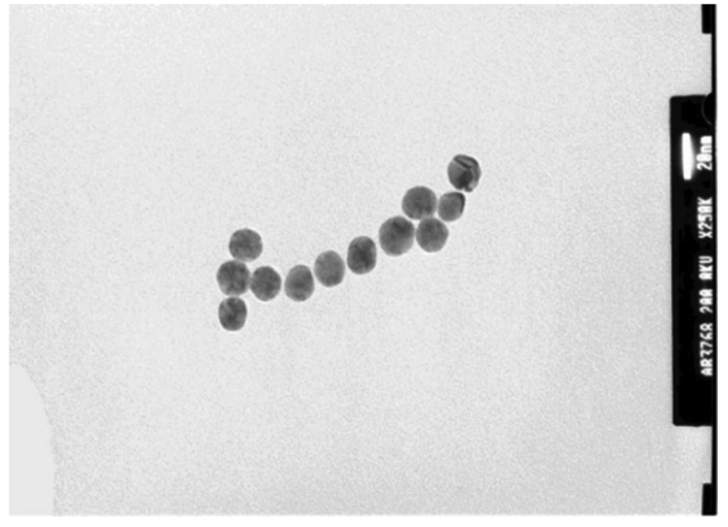

FIG. 2. TEM characterization of GNPs.

The size and shape of gold nanoparticles were identified by a scanning electron microscopy (SEM), and a transmission electron microscopy (TEM) equipped with an Energy Dispersive X-ray spectroscope (EDX). In the operation, the TEM was performed with a Jeol JEM-2010 electron microscope at a bias voltage of $200 \mathrm{kV}$. The particle size distribution in liquid was identified by a dynamic light scattering (DLS) device (Malvern nanosizer). The resulting colloid of gold nanoparticles presents in clear red-wine color and TEM images of gold nanoparticles are shown in Fig. 2. The gold nanoparticles are spherical with diameters in the range of $10 \mathrm{~nm}$ to $15 \mathrm{~nm}$. Figure 3 presents the size contribution of GNPs in DI-water, which is in the range of $10 \sim 20 \mathrm{~nm}$. A nearly full dispersed particle status in the liquid is presented in the DLS result.

\section{PROPARATION OF AGGREGATED GOLD NANOPARTICLES}

One key issue of investigating the heating effect of aggregated GNPs is to produce aggregated and dispersed GNPs of the same volume ratio. It has been widely reported that electric field can be applied for nanoparticle manipulation including electric field aided self-assembly, ${ }^{26}$ and gasphase preparation of nanoparticles. ${ }^{27}$ Here, we propose to use static electric fields to produce aggregated GNPs from mono-dispersed GNP colloids. The experiment setup is schematically depicted in Fig. 4. Two metallic electrodes were connected to a DC voltage source, and an insulator stick was bound to the bottom tips of the two electrodes to maintain a

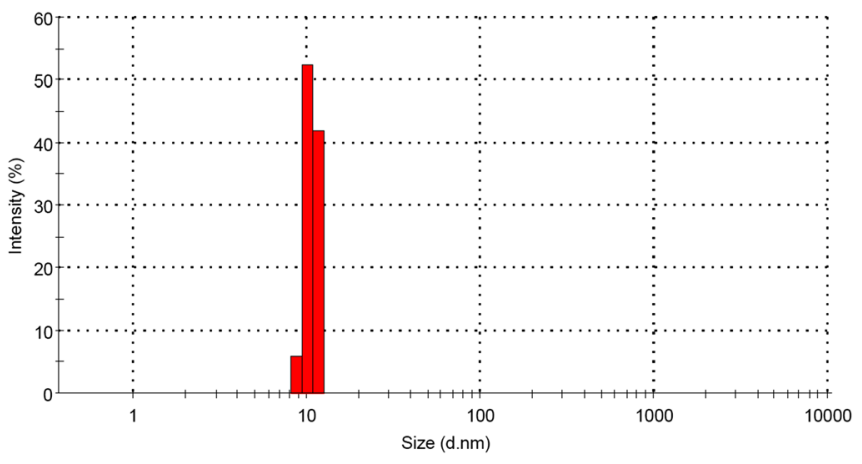

FIG. 3. Size distribution of GNPs. 


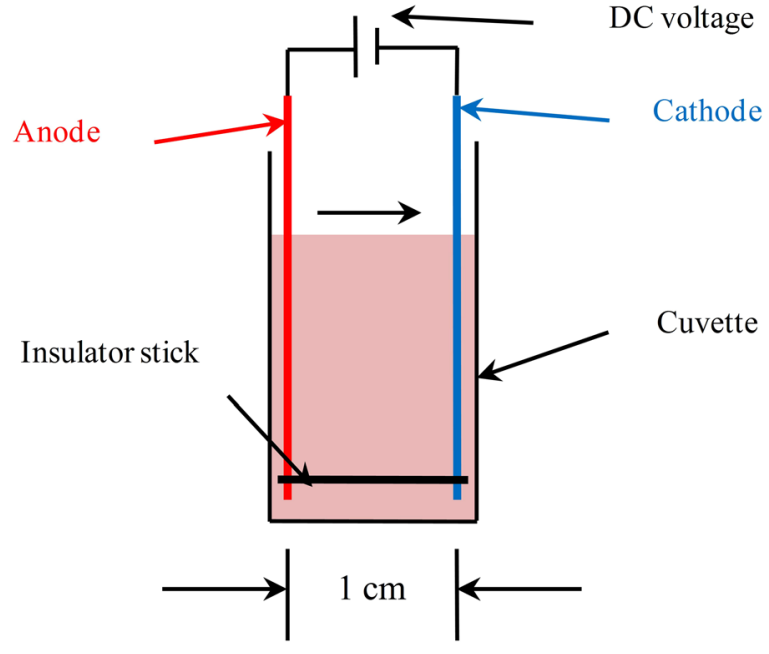

FIG. 4. Schematic diagram of applying static fields to GNP colloids to produce aggregated GNPs.

constant electric field for a given DC voltage. The distance between the two electrodes was 1 centimeter. Using $150 \mu \mathrm{mol} / \mathrm{l}$ as the original feedstock, the effect of the DC voltage and the applied duration on the agglomeration were studied, focusing on two groups: (i) $5 \mathrm{~V}$ DC voltage applied for $0,1,3,7,9 \mathrm{~min}$, and (ii) $3,7,9,11 \mathrm{~V}$ voltage for a fixed duration, i.e., $3 \mathrm{~min}$. Such an approach allows the particle volume ratio unchanged and makes it possible to compare the heating effect under the same concentration.

To characterize the performance of the agglomerations properly, several experiments were conducted, i.e., morphology examination, size distribution and UV spectroscopy measurement, and laser and RF heating. The morphology examination and size distribution of the agglomerates were conducted by SEM and DLS method. The heating effects study were carried out using a $655 \mathrm{~nm}$ red laser of $0.5 \mathrm{~W}$ and a $13.56 \mathrm{MHz}$ RF device at an output power of $600 \mathrm{~W}$.

The SEM back-scattering imaging of GNPs is shown in Fig. 5. It is seen that GNPs aggregate to form sponge-like clusters after the static electric field was applied. The morphology examination clearly shows that the loosely aggregated clusters are not solid particles. It was observed in the experiments that with the increase of electric voltage or the electrification duration, more agglomerates with large sizes were produced. With the change of morphology, the

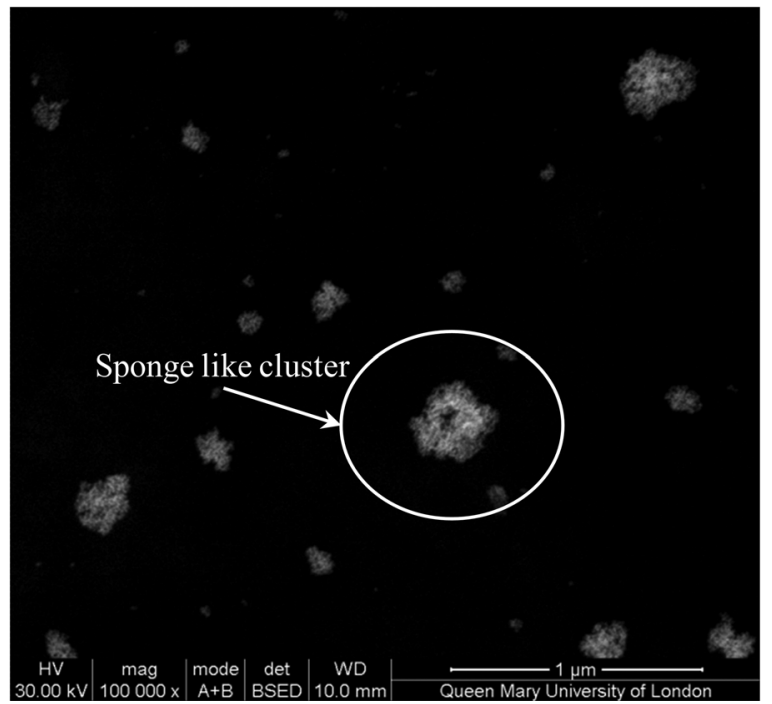

FIG. 5. SEM imaging of GNP after DC voltage of $11 \mathrm{~V}$ is applied for $3 \mathrm{~min}$. It is observed that GNPs are aggregated to form sponge-like cluster, which shows GNPs are not tightly bonded.

corresponding optical properties, such as the color index and the UV-visible absorption spectra, shall be different from those of mono-dispersed GNP colloids.

The response of GNP colloids to UV-visible light is plotted in Fig. 6(a) at a fixed voltage (5 V) but with varying electrification time. It clearly shows that after the static field is applied, the transmittance is reduced at both longer and short wavelengths. The transmittance reduction is more effective at longer electric field duration. The peak absorption wavelength, which is the spectrum corresponding to the lowest transmittance, is shown in Fig. 6(b). It shows clearly that there is a red shift as the applied electric field duration increases. Due to that the colloids contain GNPs of different size and/or GNP aggregations, the absorption become more complicated. This phenomenon will be discussed further later. From the UV-visible spectra, the color coordinates in the CIE chromaticity diagram can be deduced ${ }^{28}$ shown in Fig. 7. By locating the color coordinates, it clearly traces a trail from red towards purple with the increase of the electric field duration.

The results of the second group, i.e., the applying electric field duration is fixed but with varying voltages, are plotted in Figs. 8 and 9 for the transmission spectra and the color on the CIE chromaticity diagram, respectively. The result is similar to the first group. High voltages induce large agglomerates,

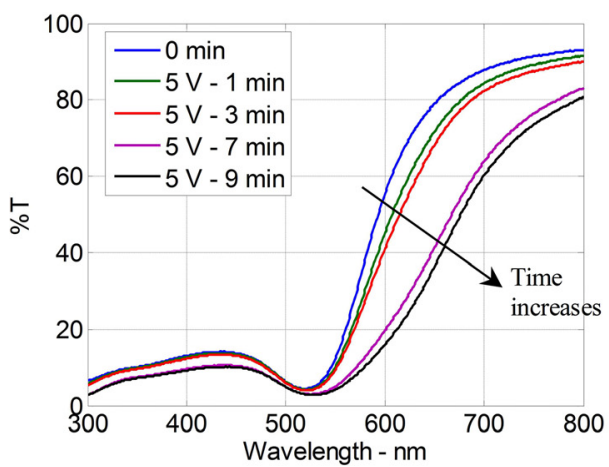

(a)

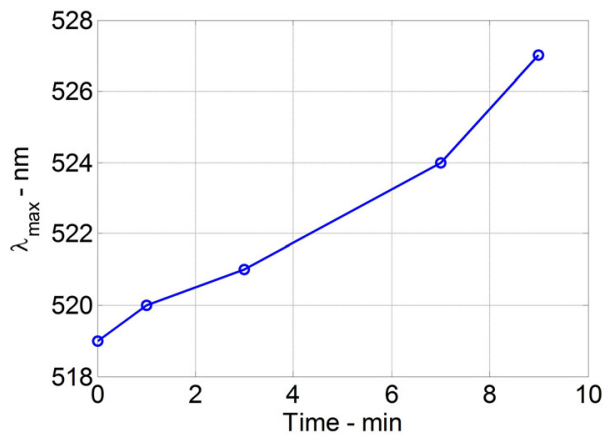

(b)
FIG. 6. (a) The UV-visible spectra of GNP colloids with DC voltage applied for different time scales; (b) The peak absorption occurs at 519, 520, 521, 524 , and $527 \mathrm{~nm}$ for the samples treated with $5 \mathrm{~V}$ DC voltage for $0,1,3$, 7 , and $9 \mathrm{~min}$. 


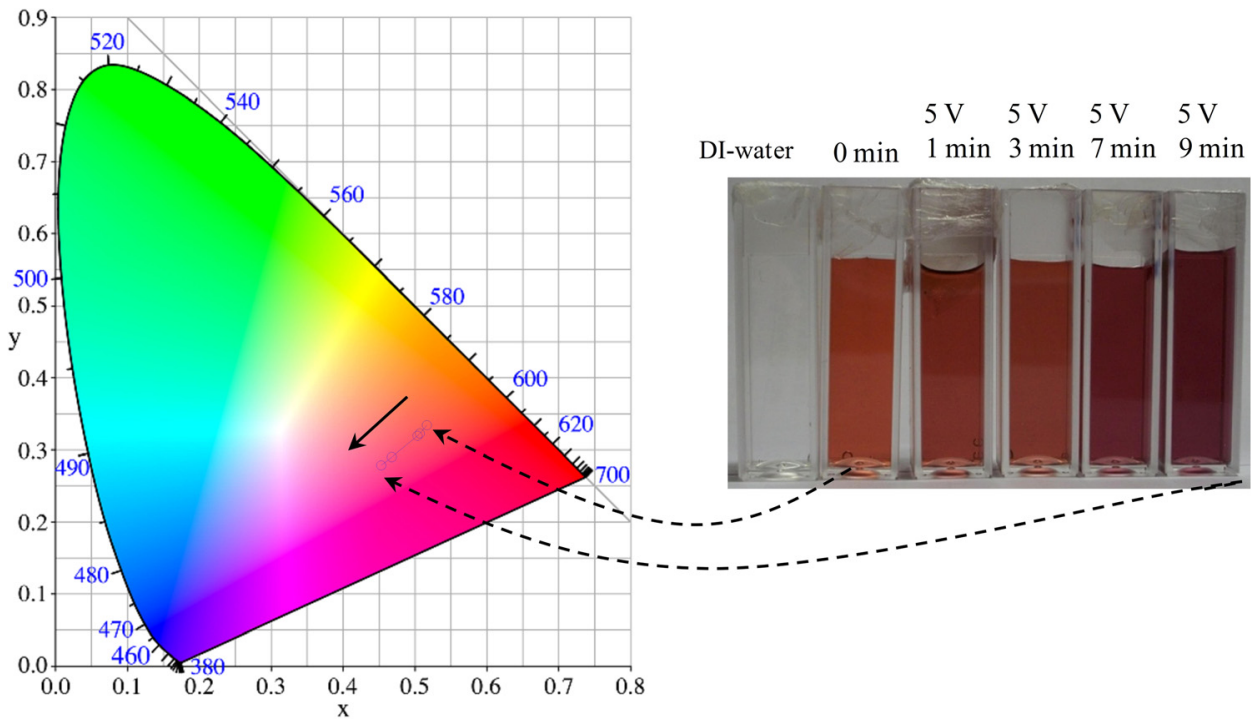

FIG. 7. Tracing the change of the chromaticity of the color of the GNP colloids. The solid arrow in the diagram indicates the direction of the change of color. The dashed curves link the GNP colloids to the color point.

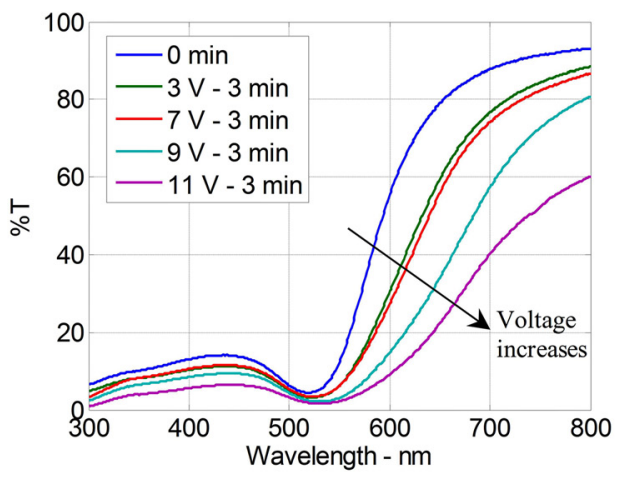

(a)

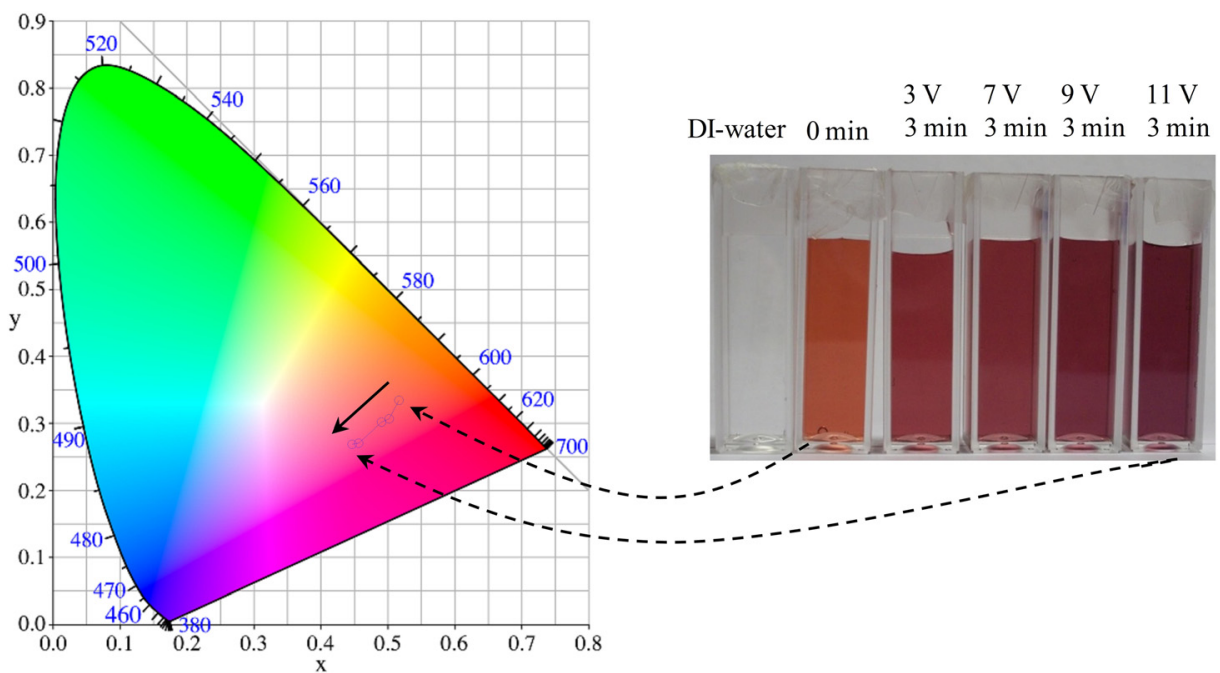

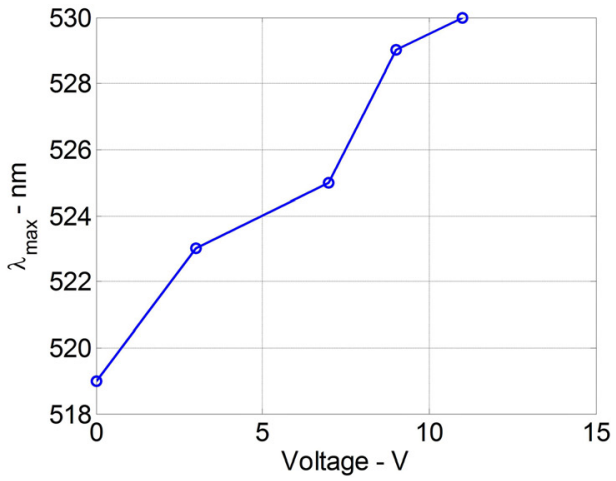

(b)
FIG. 8. The UV-visible spectra of GNP colloids with DC voltage applied for fixed time with varying voltage; (b) The peak absorption occurs at 519 , $523,525,529$, and $530 \mathrm{~nm}$ for the samples treated with $0,1,3,7,9$, and $11 \mathrm{~V}$ DC voltage for $3 \mathrm{~min}$.
FIG. 9. Tracing the change of the chromaticity of the color of the GNP colloids. The solid arrow in the diagram indicates the direction of the change of color. The dashed curves link the GNP colloids to the color point on the diagram. reduce the transmittance, and red-shift the peak absorption wavelength. The color map clearly traces a trail from red towards purple, see Fig. 9, as the voltage increases.

The size distribution of the agglomeration was measured by a Malvern Nanosizer based on the DLS method. Figure 10 presents two example diagrams of the size distribution of $5 \mathrm{~V}$ electric field applied for $1 \mathrm{~min}$, and $3 \mathrm{~min}$. Compared to the purified product shown in Fig. 3, it shows clearly that after the electric field treatment, the particle size is larger and the size range becomes much broader. The particle size appears to have two-mode distribution with double peaks, corresponding to the co-existing of small and large agglomerate.

From the experimental results of SEM, UV-visible spectroscopy and size distribution, it can be concluded that static electric field can be employed to produce loosely aggregated GNPs. It has to be noted that the aggregated GNPs are not solid ones, as evidenced by the SEM imaging and UV-spectroscopy. Actually, the surface Plasmon resonance 


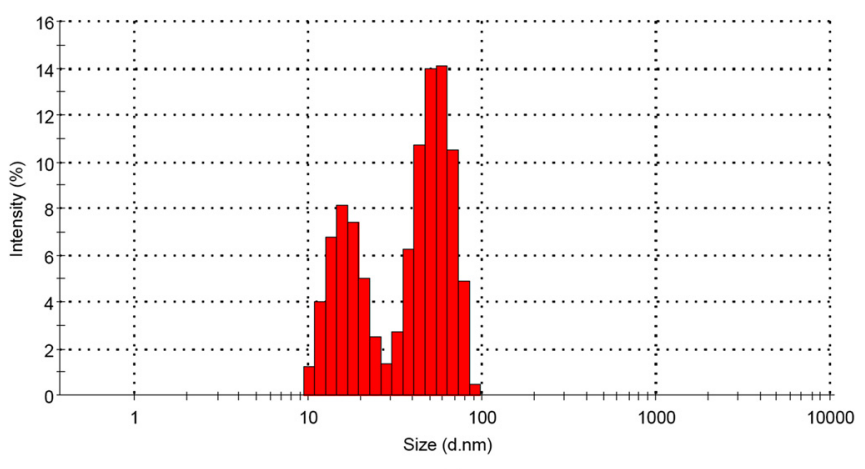

(a)

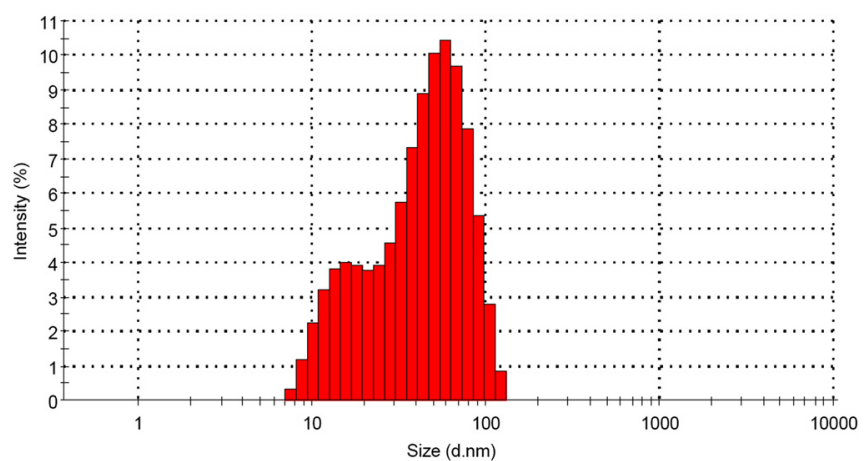

(b)

FIG. 10. Size distribution of (a) $5 \mathrm{~V}-1 \mathrm{~min}$, (b) $5 \mathrm{~V}-3 \mathrm{~min}$.

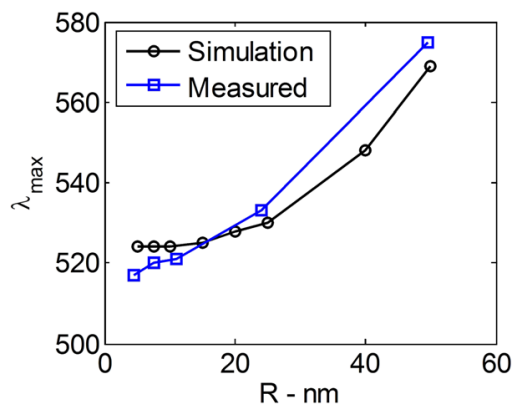

FIG. 11. The wavelength where the extinction efficiency peaks against the radius (the measured data are adapted from Refs. 30-32).

(SPR) wavelength for $100 \mathrm{~nm}$ GNPs shall be much longer than that in Figs. 6(b) and 8(b). For instance, the SPR wavelength is over $545 \mathrm{~nm}$ when the radius of GNP is $40 \mathrm{~nm}$ $(\mathrm{D}=80 \mathrm{~nm})$, see Fig. 11. The corresponding SPR wavelength to Figs. 10(a) and 10(b) is $524 \mathrm{~nm}$. The difference may be attributed to (i) the wide particle size distribution, as shown in Fig. 10, and (ii) different scattering characteristics of spongelike agglomerates and the solid particles. The SEM image shows that aggregated GNPs are loosely bonded, whose scattering effect shall behave differently to solid GNPs of the same size. Understanding the scattering properties of loosely aggregated GNPs is still presenting as a challenge task. One possible approach is to use numerical method such as the optical immersed Finite-difference time domain (FDTD) method. ${ }^{29}$ Nevertheless, it has to be stressed that loosely aggregated GNPs are of practical significance, since they resemble the scenario where tissues are incubated with GNPs.

\section{BULK HEATING EFFECT BY A 655 nm LASER}

The experiment setup for the laser heating is shown in Fig. 12. The samples were placed in the chamber of a microwave oven with an opening on the top ceiling, where a $655 \mathrm{~nm}$ red light laser was aligned to the center of the cuvette. The transient temperature was measured by an optical fiber sensor of an accuracy of $\pm 0.1^{\circ} \mathrm{C}$. The optical fiber was attached to the inner wall of the cuvette to avoid direct laser illumination, and with careful alignment to ensure that the location of the measurement position was the same for all the samples. The power of the laser was $0.5 \mathrm{~W}$, which was uniformly spread in a rectangular spot size of $1 \mathrm{~cm}$ in length and $0.5 \mathrm{~cm}$ in width, smaller than the size of the cuvette, i.e., $1.25 \mathrm{~cm}$ by $1.25 \mathrm{~cm}$. The volume of each sample was controlled at $4.0 \pm 0.1 \mathrm{ml}$. Before the measurement, all samples were placed in the chamber of the microwave oven to achieve a thermal equilibrium. The transient bulk temperature is plotted in Fig. 13(a), and Fig. 13(b), for the first group and the second group of samples, respectively.

The results clearly show that much higher temperature rise can be observed after the electric field treatment. For instance, comparing with the mono-dispersed GNPs (i.e., $0 \mathrm{~V}$ ), the bulk temperature was increased by $\sim 1 / 3$ comparing for $5-\mathrm{V}$ treatment of $9 \mathrm{~min}$. Either increasing the voltage or increasing the electric field applying duration can increase the bulk temperature appreciably, which is related to the particles' morphology change.

\section{BULK HEATING EFFECT BY A 13.56 MHz RF FIELD}

The device of delivering $13.56 \mathrm{MHz} \mathrm{RF}$ power is schematically shown in Fig. 14, where a microstrip waveguide
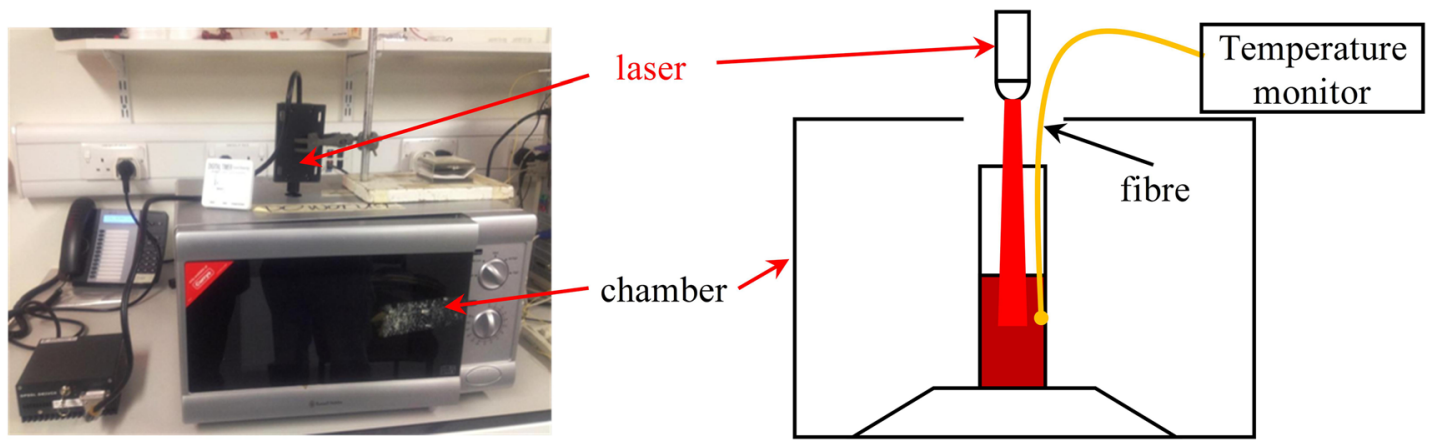

FIG. 12. Experiment setup for heating effect study. A cuvette is placed in the chamber of a microwave oven; the $655 \mathrm{~nm}$ red laser is aligned to GNP colloids. 


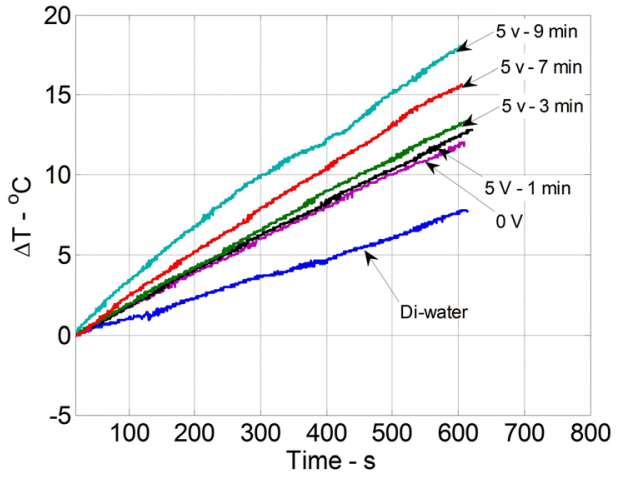

(a)

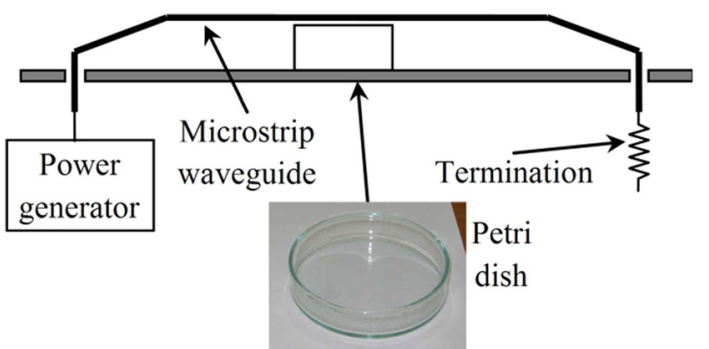

FIG. 14. Schematic diagram of the RF heating system. Reproduced by permission from Liu et al., Nanoscale 4, 3945-3953 (2012). Copyright 2012 by The Royal Society of Chemistry.

was fed by a coaxial line connecting to a $600 \mathrm{~W}$ power generator. The other end of the microstrip waveguide was terminated by a $50 \Omega$ load. A Petri dish was placed in the $10 \mathrm{~mm}$ high air gap between the two conducting plates of the microstrip waveguide. The field distribution of this structure was

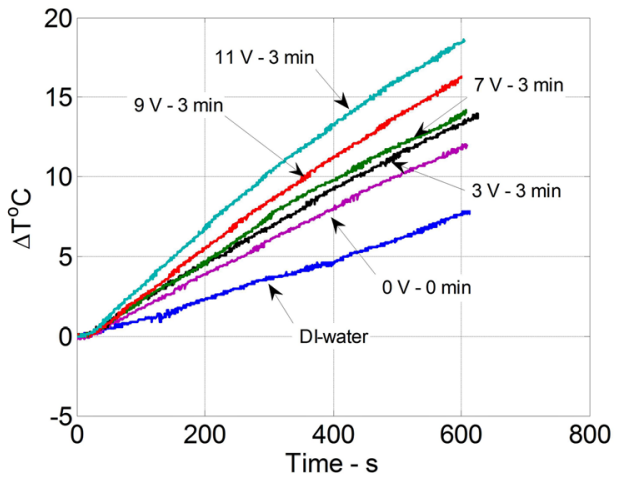

(b)
FIG. 13. The heating effect of DI-water, GNP colloids without static field treatment and GNPs colloids treated with DC voltage at (a) $5 \mathrm{~V}$ for 1, 3, 7, 9 min; (b) 3, 7, 9, $11 \mathrm{~V}$ for $3 \mathrm{~min}$. The laser is working at $655 \mathrm{~nm}$, full power $0.5 \mathrm{~W}$. The measurement accuracy is $\pm 0.1^{\circ} \mathrm{C}$.

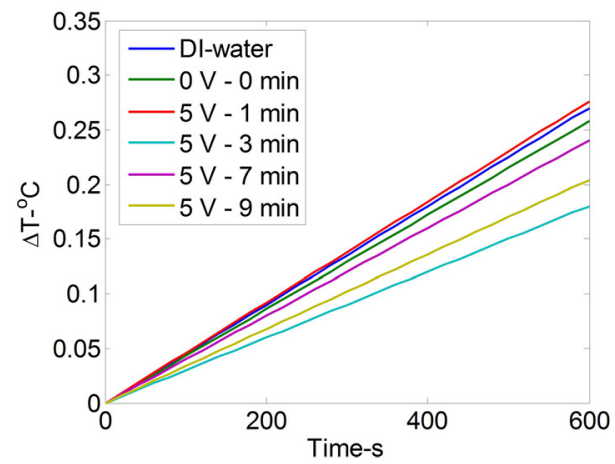

(a)

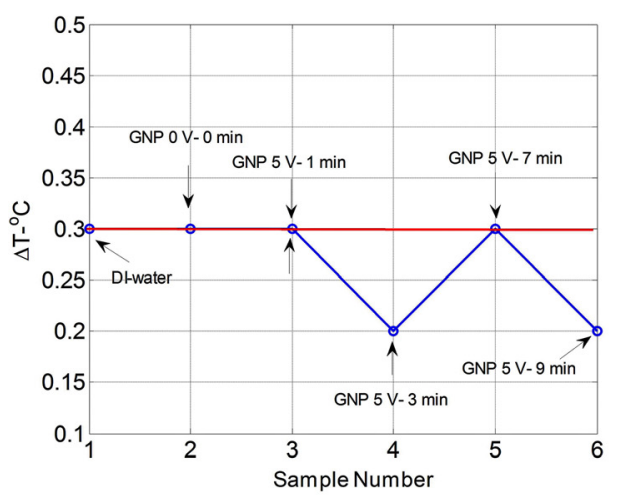

(c)

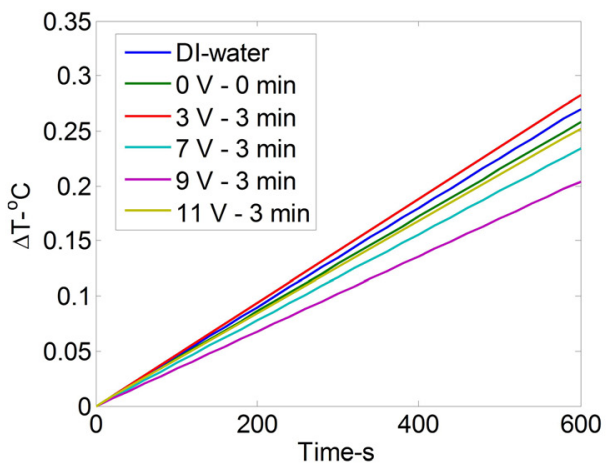

(b)

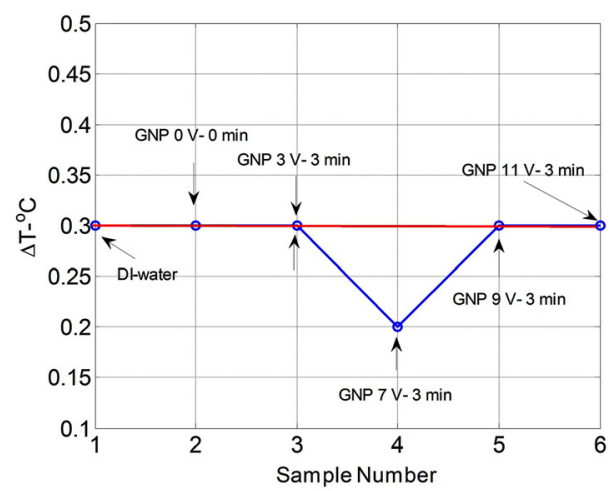

(d)
FIG. 15. Heating of GNP colloids after DC voltage is applied, the measurement accuracy is $\pm 0.1^{\circ} \mathrm{C}$. (a) Fitting results for $5 \mathrm{~V}$ with varying time period; (b) Fitting results for varying voltage and fixed time period; (c) Final temperature rise for $5 \mathrm{~V}$ with varying time period; (d) Final temperature rise for varying voltage and fixed time period. 
placing any sample and found that within $10 \mathrm{~min}$, the average temperature rise for 10 measurements was within $0.3 \pm 0.1^{\circ} \mathrm{C}$, which implies that any temperature rise within $0.3 \pm 0.1^{\circ} \mathrm{C}$ shall not be decisively attributed to the heating effect of the samples.

\section{DISCUSSION}

From the heating results of both $655 \mathrm{~nm}$ red light laser and $13.56 \mathrm{MHz} \mathrm{RF}$ field, it can be seen that aggregated GNPs can enhance the heating effect at $655 \mathrm{~nm}$, while no observable enhancement is found at $13.56 \mathrm{MHz}$. Such a significant different behavior between two frequencies requires further prudent investigation.

The significant temperature rise in the visible light spectrum can be attributed to a combined effect of reduced transmittance and a stronger surface Plasmon effect. The UV-visible spectroscopy results, Figs. 6(a) and 8(a), already show that the transmittance of static electric field treated GNPs colloid is decreased significantly compared with that of mono-dispersed GNPs. For instance, the transmittance of the sample is decreased from $\sim 80 \%$ to $\sim 40 \%$ at $655 \mathrm{~nm}$ after the $5 \mathrm{~V}-9 \mathrm{~min}$ treatment, which results in a significant increase in the absorption rate that contribute to the temperature rise. The SPR wavelength of GNPs $10 \sim 20 \mathrm{~nm}$ in diameter is around $520 \mathrm{~nm}$. A $655 \mathrm{~nm}$ red light laser can still produce some heating effect when illuminating on these GNP colloids. The red-shift of the agglomerated particles, Figs. 6(b) and 8(b), will certainly increase the heating effect further. Physically it is not well understood why aggregated GNPs can increase the absorption of light. Other simulation results suggested that dimer-structured silver nanoparticles $^{34-36}$ could enhance the electric field, whereas

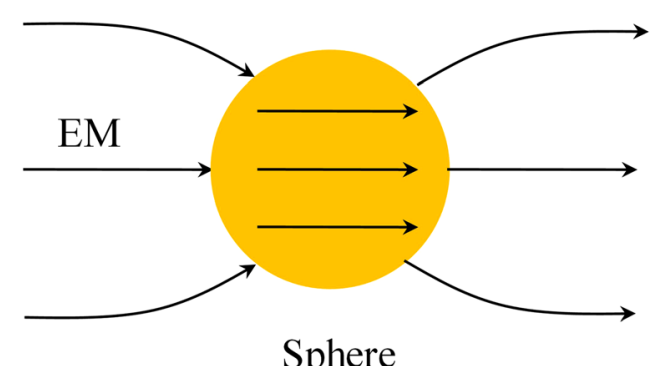

FIG. 16. Field distribution of a spherical particle in a uniform field.

the effect was much stronger for a trimer or quadrumer structure. The loosely aggregated GNPs resemble the arrangement of dimer/trimer/quadrumer structure, yet with more particles, which would produce a similar electric field enhancement effect. It is likely that this enhancement of electric field could contribute to the increased absorption of electromagnetic energy, hence an increased heating effect. Clearly such a hypothesis requires further theoretical and simulation verification.

However, for $13.56 \mathrm{MHz} \mathrm{RF}$, aggregated GNPs do not show any heating effects compared with mono-dispersed GNP colloids, which can be explained by the classical Joule heating model due to an insignificant electric field enhancement effect. In the literature, the heating of GNPs by RF fields has been subjecting to intensive discussion recently. For uncoated neutral GNPs, the heating mechanisms have been discussed extensively in Hanson's work. ${ }^{15,17}$ For simplicity, the following model is used to estimate the heating effect in a order of magnitude manner.

For spherical particles, the field distribution inside the particles, shown in Fig. 16, can be written as ${ }^{37}$

$$
\vec{E}(\vec{r})=\left\{\begin{array}{c}
\hat{r} E_{0}\left(-1-\frac{\varepsilon-\varepsilon_{1}}{\varepsilon+2 \varepsilon_{1}} \cdot \frac{2 R^{3}}{r^{3}}\right) \cos \theta+\hat{\theta} E_{0}\left(1-\frac{\varepsilon-\varepsilon_{1}}{\varepsilon+2 \varepsilon_{1}} \cdot \frac{2 R^{3}}{r^{3}}\right) \sin \theta \quad r>R \\
-\hat{z} E_{0} \frac{3 \varepsilon_{1}}{\varepsilon+2 \varepsilon_{1}} \quad 0 \leq r<R
\end{array},\right.
$$

where $E_{0}$ is the incident electric field strength, $\varepsilon_{1}$ and $\varepsilon$ are the relative permittivities of the base fluid and the particles, respectively, and $R$ is the particle radius. The heating effect due to the particles can be written as

$$
\Delta T=\frac{4}{3} \pi R^{3} \cdot N \cdot \frac{\sigma_{e f f}|E|^{2}}{C \rho V} \cdot t=r_{V} \cdot \sigma_{e f f}|E|^{2} \cdot t,
$$

where $\Delta T$ is the bulk temperature rise, $N$ is the number of particles, $\sigma_{e f f}$ is the effective conductivity, $C$ is the specific heat capacity, $\rho$ is the mass density, $V$ is the volume of the sample, and $t$ is the heating time. As the volume ratio of GNP can be written as

$$
r_{V}=\frac{4 \pi R^{3}}{3 V} \cdot N
$$

The bulk temperature rise can be written consequently as

$$
\Delta T=r_{V} \cdot \sigma_{e f f}|E|^{2} \cdot t=r_{V} \cdot \frac{9 \omega \varepsilon_{0} \varepsilon^{\prime \prime} \varepsilon_{1}^{2}\left|E_{0}\right|^{2}}{\left(\varepsilon^{\prime}+2 \varepsilon_{1}\right)^{2}+\varepsilon^{\prime \prime 2}} \cdot t
$$

by noting that $\varepsilon=\varepsilon^{\prime}-j \varepsilon^{\prime \prime}$ and $\sigma_{e f f}=\omega \varepsilon_{0} \varepsilon^{\prime \prime}$. In addition, due to the fact of that water is a low loss medium at $13.56 \mathrm{MHz}$, $\varepsilon_{1}$ can be considered as a real number. By the Drude model, the conductivity of a metallic material is

$$
\sigma=\frac{\sigma_{0}}{(1+j \omega \tau)}
$$

where $\sigma_{0}$ is the DC conductivity, and $\tau$ is the relaxation time. In consequence, the relative permittivity of the metallic material can be written as 


$$
\varepsilon=1-j \frac{\sigma_{0}}{\omega \varepsilon_{0}(1+j \omega \tau)}
$$

For gold particles, $\sigma_{0}$ is around $4.25 \times 10^{7} \mathrm{~S} / \mathrm{m}$, and $\tau$ is on the order of $10^{-14} \mathrm{~s} .{ }^{38}$ The relative permittivity can be approximated as

$$
\varepsilon=1-j \frac{\sigma_{0}}{\omega \varepsilon_{0}},
$$

and consequently

$$
\begin{aligned}
\Delta T & =r_{V} \cdot \sigma_{e f f}|E|^{2} \cdot t \approx r_{V} \cdot \frac{9 \sigma_{0} \varepsilon_{1}^{2}\left|E_{0}\right|^{2}}{\left(1+2 \varepsilon_{1}\right)^{2}+\left(\frac{\sigma_{0}}{\omega \varepsilon_{0}}\right)^{2}} \cdot t \\
& <5 r_{V} t \times 10^{-10} \approx 10^{-10 \circ} \mathrm{C} .
\end{aligned}
$$

The volume ratio of GNP is usually much smaller, i.e., in the order of a few ppms for most practical applications. Therefore, the temperature rise in $10 \mathrm{~min}$ is very small. For aggregated nanoparticles, the temperature rise $\Delta T_{a}$ over $\Delta T$

$$
\frac{\Delta T_{a}}{\Delta T} \propto \frac{\left|E_{a}\right|^{2}}{|E|^{2}} .
$$

In order to observe noticeable increase in heating effect, the enhancement of electric field should be on the order of $10^{5}$, which has not been observed yet. Such an effect is understandable as the periphery dimension of nanoparticles is much smaller than the wavelength of $13.56 \mathrm{MHz}$ RF field (more than $22 \mathrm{~m}$ ). Even the aggregated nanoparticles, say 100 micrometer in diameter, are still too small to produce any effect within the accuracy of measurement. However the phenomenon in the visible light region is different as the plasomonic resonance effect may take place, which can produce a stronger heating effect.

\section{CONCLUSION}

This work investigated the bulk heating effect of agglomerated gold nanoparticle (GNP) colloids under a $655-\mathrm{nm}$ laser and a $13.56 \mathrm{MHz}$ RF electric field. A novel method using static electric field was developed to produce aggregated GNPs and characterized by the UV-visible spectroscopy, dynamic light scattering, SEM and CIE chromaticity diagram. With the application of the electric field, loose agglomerates were formed, whose effect increased with the increase of the electric voltage or the increase of the electric treatment time. The heating results showed that at the wavelength of $655 \mathrm{~nm}$, that aggregated GNPs could enhance the bulk heating effect significantly, which is associated with the reduced transmittance effect and an increased surface Plasmon resonance effect. However, at $13.56 \mathrm{MHz}$, the RF heating of aggregated GNPs did not produce any observable heating enhancement within the measurement accuracy, which can be explained by the classical Joule heating model due to an insufficient increase in the electric field. Such a result disproved the suggestions that the pure agglomerated particles could produce significant heating effect at 13.56 MHz RF field.

\section{ACKNOWLEDGMENTS}

This research was partly supported by the Scientific Research Foundation for the Returned Overseas Chinese Scholars, State Education Ministry, the Fundamental Research Funds for the Central Universities of China, and the National Science Foundation of China through its Grant No. 51228601.

${ }^{1}$ E. Hutter and D. Maysinger, Microsc. Res. Tech. 74, 592 (2011).

${ }^{2}$ E. Petryayeva and U. J. Krull, Langmuir 28, 13943 (2012).

${ }^{3}$ J. Cheng, Y. J. Gu, S. H. Cheng, and W. T. Wong, J. Biomed. Nanotechnol. 9, 1362 (2013).

${ }^{4}$ R. Khandelia, A. Jaiswal, S. S. Ghosh, and A. Chattopadhyay, Small 9, 3493 (2013).

${ }^{5}$ L. Minai, D. Yeheskely-Hayon, and D. Yelin, Sci. Rep. 3, 2146 (2013).

${ }^{6}$ S. Jung, J. Nam, S. Hwang, J. Park, J. Hur, K. Im, N. Park, and S. Kim, Anal. Chem. 85, 7674 (2013).

${ }^{7}$ C. J. Gannon, C. R. Patra, R. Bhattacharya, P. Mukherjee, and S. A. Curley, J. Nanobiotechnol. 6, 2 (2008).

${ }^{8}$ J. Cardinal, J. R. Klune, E. Chory, G. Jeyabalan, J. S. Kanzius, M. Nalesnik, and D. A. Geller, Surgery 144, 125 (2008).

${ }^{9}$ C. H. Moran, S. M. Wainerdi, T. K. Cherukuri, C. Kittrell, B. J. Wiley, N. W. Nicholas, S. A. Curley, J. S. Kanzius, and P. Cherukuri, Nano Res. 2, 400 (2009).

${ }^{10}$ D. E. Kruse, D. N. Stephens, H. A. Lindfors, E. S. Ingham, E. E. Paoli, and K. W. Ferrara, IEEE Trans. Biomed. Eng. 58, 2002 (2011).

${ }^{11}$ E. S. Glazer, C. Zhu, K. L. Massey, C. S. Thompson, W. D. Kaluarachchi, A. N. Hamir, and S. A. Curley, Clin. Cancer Res. 16, 5712 (2010).

${ }^{12}$ E. S. Glazer, K. L. Massey, C. Zhu, and S. A. Curley, Surgery 148, 319 (2010).

${ }^{13}$ D. Li, Y. S. Jung, S. Tan, H. K. Kim, E. Chory, and D. A. Geller, J. Colloid Interface Sci. 358, 47 (2011).

${ }^{14}$ X. Liu, H.-J. Chen, X. Chen, C. Parini, and D. Wen, Nanoscale 4, 3945 (2012).

${ }^{15}$ G. W. Hanson and S. K. Patch, J. Appl. Phys. 106, 054309 (2009).

${ }^{16}$ G. Speit and P. Schütz, Mutat. Res. 747-748, 1 (2013).

${ }^{17}$ G. W. Hanson, R. C. Monreal, and S. P. Apell, J. Appl. Phys. 109, 124306 (2011).

${ }^{18}$ E. Sassaroli, K. C. P. Li, and B. E. O’Neill, J. Phys. D: Appl. Phys. 45, 075303 (2012).

${ }^{19}$ S. J. Corr, M. Raoof, Y. Mackeyev, S. Phounsavath, M. A. Cheney, B. T. Cisneros, M. Shur, M. Gozin, P. J. McNally, L. J. Wilson, and S. A. Curley, J. Phys. Chem. C 116, 24380 (2012).

${ }^{20}$ R. S. McCoy, S. Choi, G. Collins, B. J. Ackerson, and C. J. Ackerson, ACS Nano 7, 2610 (2013).

${ }^{21}$ B. H. San, S. H. Moh, and K. K. Kim, Int. J. Hyperthermia 29, 99 (2013).

${ }^{22}$ H. K. Kim, G. W. Hanson, and D. A. Geller, Science 340, 441 (2013).

${ }^{23}$ W. A. Curtin, R. C. Spitzer, N. W. Aschcroft, and A. J. Sievers, Phys. Rev. Lett. 54, 1071 (1985).

${ }^{24}$ B. P. Devaty and A. J. Sievers, Phys. Rev. B 41, 7421 (1990).

${ }^{25}$ H.-J. Chen and D. Wen, Nanoscale Res. Lett. 6, 198 (2011).

${ }^{26}$ J. Park and W. Lu, Appl. Phys. Lett. 93, 243109 (2008).

${ }^{27}$ M. T. Swihart, Curr. Opin. Colloid Interface Sci. 8, 127 (2003).

${ }^{28}$ B. J. Clark, T. Frost, and M. A. Russell, UV Spectroscopy: Techniques, Instrumentation and Data Handling (Chapman \& Hall, London, 1993).

${ }^{29}$ S. Tanev, V. V. Tuchin, and P. Paddon, J Biomed Opt. 11, 064037 (2006).

${ }^{30}$ E. Hutter and J. Fendler, Adv. Mater. 16, 1685 (2004).

${ }^{31}$ M. Homberger and U. Simon, Philos. Trans. R. Soc., A 368, 1405 (2010).

${ }^{32}$ J. Zhang, T. Atay, and A. V. Nurmikko, Nano Lett. 9, 519 (2009).

${ }^{33}$ X. Liu, H.-J. Chen, Y. Alfadhl, X. Chen, C. Parini, and D. Wen, J. Appl. Phys. 113, 074902 (2013).

${ }^{34}$ E. Hao and G. C. Schatz, J Chem. Phys. 120, 357 (2004).

${ }^{35}$ K. Kim, D. Shin, K. L. Kim, and K. S. Shin, Phys. Chem. Chem. Phys. 12, 3747 (2010).

${ }^{36}$ S. Campione, S. M. Adams, R. Ragan, and F. Capolino, Opt. Express 21, 7957 (2013).

${ }^{37}$ J. D. Jackson, Classical Electrodynamics (John Wiley \& Sons Inc., New York, 1975).

${ }^{38}$ C. Kittel, Introduction to Solid State Physics (John Wiley \& Sons, Inc., New York, 1996). 